\title{
ENERGY-SAVING OPERATION OF MULTISTAGE STOCHASTIC MANUFACTURING SYSTEMS BASED ON FUZZY LOGIC
}

\author{
Wang, J. F. ; Fei, Z. C. ${ }^{*}$; Chang, Q. ${ }^{* *}$; Fu, Y. ${ }^{*} \&$ Li, S. Q. ", \\ * Department of Industrial and Manufacturing System Engineering, Huazhong University of Science \\ and Technology, Wuhan 430074, China \\ ** Department of Mechanical and Aerospace Engineering, University of Virginia, Charlottesville, \\ Virginia 22904, USA \\ E-Mail: wangjf@hust.edu.cn, feizicheng_hust@163.com,qc9nq@virginia.edu, \\ laura_fy@mail.hust.edu.cn, sqli@hust.edu.cn $\left({ }^{\#}\right.$ Corresponding author)
}

\begin{abstract}
The energy-saving operation of manufacturing systems is becoming increasingly significant due to the pressures of social sustainability and cost reduction. One method of saving energy in manufacturing systems is reducing the idle times of machines, which are non-value-added activities but consume non-negligible energy. Based on real-time data of manufacturing systems, this paper proposes a fuzzy control method to switch a machine into sleep/off mode when the idle state is about to arise and turn the machine on when production has to be resumed. A basic control module of a manufacturing system is described, considering stochastic failure of machines. The fuzzy rules for energy-saving operation are presented by analysing the buffer levels of machines based on production knowledge. Simulation studies are carried out to validate and illustrate the effectiveness of the method both for serial and parallel manufacturing systems. The proposed fuzzy control method is a simple and preferable way to achieve the energy-saving operation of unreliable manufacturing systems.

(Received, processed and accepted by the Chinese Representative Office.)
\end{abstract}

Key Words: Energy-Saving Operation, Fuzzy Logic, Multistage Manufacturing System

\section{INTRODUCTION}

The manufacturing industry has long been the largest energy consumer and greenhouse gas emitter. Manufacturing activities account for $33 \%$ of the total energy consumption and lead to about $38 \%$ of industrial energy-related emissions of carbon dioxide. The pressures resulting from high energy costs, environmental rules, and the consuming behaviour of customers have caused energy-conscious manufacturing operations to gain more and more attention all over the world [1].

Manufacturing systems are complex dynamic systems with interdependence among machines and buffers, so properly timing manufacturing tasks is difficult. Unscheduled downtimes result in the transition of machine operational states. It is usual to see some machines standing idle during a production process. Decreasing idle periods could significantly improve eco-efficiency if the idle machines were turned off [2]. By investigating several standby states on production equipment with known short periods of all nonproductive time, Weinert and Mose [3] showed that about $50 \%$ to $60 \%$ of energy consumption could be reduced compared with the reference case. A networked, digital, and smart vision of manufacturing systems was described in Industry 4.0, where Internet of Things technologies monitored production processes in almost real time [4]. The machine state, the real-time buffer level, and the energy consumption of equipment can be sensed. All these pieces of production information provide operators more opportunities to conduct energy-saving operation in a sensing, smart, and sustainable manufacturing enterprise [5]. 
Product-machine scheduling and real-time controls of machine state during production are two main methods used in the energy-saving operation of manufacturing systems. In scheduling research, energy consumption was considered as one optimization parameter [6]. Multi-objective optimization problems were formalized, and better task sequences were searched by using optimization algorithms [7]. The scheduled product order combined with the periodical shutdown of machines decreased the idle status to minimize energy consumption. Because different machines had different levels of reliability, random failures were rarely considered in scheduling algorithms. Instead of scheduling products and machines, the machine state control method for energy-efficient operation makes a suitable ON/OFF decision via policies or an analytical model based on real-time production data.

Quantitative policies can be defined to adjust machine states for energy-saving operation. Nine switch-off dispatching policies were studied to minimize energy consumption for a onebuffer, one-machine system [8]. It was found that energy savings reached $80 \%$ when a nonbottleneck machine was turned off. By combining an M/M/1 model and serial production lines with an energy control policy, Prabhu et al. [9] switched machines into a low-power idle mode when the idle time exceeded the defined threshold value. But throughput was not analysed when the policy was applied. For a single machine visited by a single part type with stochastic inter-arrival times, Frigerio et al. $[10,11]$ presented five control policies to switch the machine off and on for energy saving according to the buffer level, where the warm-up state was not considered. For Bernoulli serial lines, Jia et al. [12] studied the system performances, e.g., production rate and power, by defining six switch-on/off rules of a machine based on buffer occupancy. The disadvantage of the policy strategy is that the parameters of the policies, e.g., buffer occupancy and idle time threshold, are not easy to predefine for unreliable machines in a manufacturing system.

The analytical method was also used to control the ON/OFF states of machines to save energy. Chang et al. [13] investigated the energy-saving windows by analysing the random disturbing events in a serial automotive manufacturing line. Zou et al. [14] developed a stochastic model of the downtime opportunity to estimate the opportunity windows and recovery times of machines. They also integrated the current and predicted system performance to improve the overall profit and energy efficiency [15]. For a multistage serial and parallel manufacturing system, a control algorithm for the energy-saving opportunity window was proposed to improve the system's energy efficiency through event-based analysis [16]. The above method was only applied to an asynchronous line. Sun and Li [17] discussed single machine control for energy-saving opportunity in a serial line. They also adopted the Markov decision process to describe the interaction among several hibernation states and energy control decisions [18]. An approximate dynamic programming algorithm was proposed to get a near-optimal state evolution of a machine for energy-saving operation. $\mathrm{Su}$ et al. [19] developed an integrated model to minimize energy consumption while maintaining the desired productivity in Bernoulli serial lines with unreliable machines and finite buffers. Usually, the analytical methods had some strict assumptions about the manufacturing system, such as machine reliability type, serial/asynchronous line, or single machine control.

Current policy strategies and analytical methods try to quantitatively decide when the energy-saving operation should be taken and how long the energy-saving operation should last. As an effective method of manufacturing system control in production planning, scheduling, and process, fuzzy logic provides a good way to integrate human knowledge into the control cycle and allow a system to work with imprecise and incomplete information [20-23]. This paper is an extension of our work originally presented in [24], where a fuzzy decision method was proposed to decrease the duration of idle states by switching machines OFF/ON based on real-time production line data. By extending the basic modules of 
manufacturing systems, the fuzzy energy control method was efficiently used in a production line with disassembly and assembly stations [25]. In this paper, a basic control module for energy control of serial and parallel lines are discussed in detail. Simulations are carried out to evaluate system performances, i.e., throughput and energy consumption, and discussions are presented. Compared with quantitative policy strategies and analytical models, our method can be used in both serial and parallel lines without the restriction on machine reliability. Single machine or multi-machine control can be flexibly realized. The proposed fuzzy system is computationally simple, thereby facilitating its application to real-time control of manufacturing systems.

\section{FUZZY CONTROL MODULE FOR ENERGY-SAVING OPERATION OF MANUFACTURING SYSTEMS}

\subsection{Basic control module for energy saving of manufacturing system}

In this paper, a basic control module for one machine with two connected buffers (1M2B), which is described in Fig. 1, is used as the basis to construct an energy-saving operation scheme for complex manufacturing systems. In our study, discrete time is used in the production process.

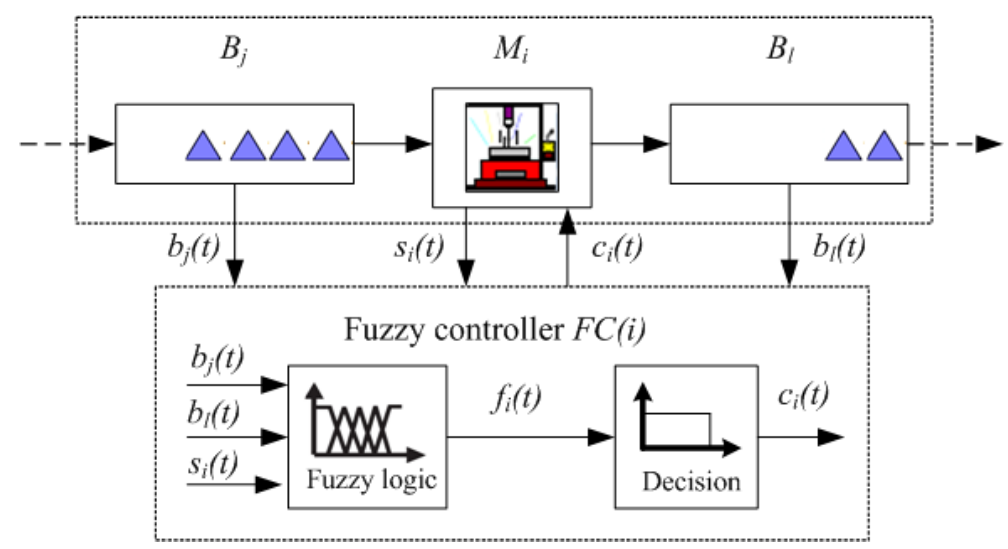

Figure 1: Basic control module of manufacturing system.

For a general multistage serial or parallel manufacturing system, the following assumptions are made:

a) Machine $M_{i}(i=1,2, \ldots, n)$ has a constant cycle time $\tau_{i}$. The cycle times of machines can be the same or different.

b) Buffers $B_{j}$ and $B_{l}(j, l=1,2, \ldots, m$ and $j<l)$ are characterized by their maximum capacity $B C$. Each buffer has a real-time buffer level $\left(b_{j}(t)\right.$ and $\left.b_{l}(t)\right)$ at each discrete time slot.

c) The mean time between failure $(M T B F)$ and mean time to repair (MTTR) of machines are assumed to follow a known probability distribution.

d) At the beginning of a time slot, $M_{i}$ is starved if it is powered on, and its upstream buffer $B_{j}$ is empty. It is assumed that the first machine is never starved.

e) At the beginning of a time slot, $M_{i}$ is blocked if it is powered on, and its downstream buffer $B_{l}$ is full. It is assumed that the last machine is never blocked.

f) A machine in power-on state will consume energy, such as electricity, fuel, gas, and compressed air. All the consumed material will be converted into energy.

g) Machine states include work state, normal idle state, failure state, energy-saving state (standby state or power off state), and warm-up state (from failure state or from energysaving state to work state). Different machine states consume respective energy. A machine in shutdown or failure mode does not consume energy. 
h) Buffers do not consume energy.

In Fig. 1, the fuzzy controller of the 1M2B module has two decision phases. The first phase is fuzzy reasoning with output $f_{i}(t)$,and the second phase is a threshold comparison with output $c_{i}(t)$.A commonly used fuzzy inference method, the Mamdani method [26], is applied in the first phase. The input variables of a basic module at each expected time slot include $b_{j}(t), b_{l}(t)$, and $s_{i}(t)$, which are the real-time level of upstream buffer $B_{j}$, the real-time level of downstream buffer $B_{l}$, and the current up/down state of machine $M_{i}$, respectively.

During the production process, the real-time level of a buffer, i.e., $b_{j}(t)$ and $b_{l}(t)$, takes linguistic variables with defined membership functions. In this paper, the linguistic value of buffer levels is taken from the set $B L=\{$ Empty, Almost Empty, Normal, Almost Full, Full $\}$. Triangle and trapezoid membership functions are commonly used as the linguistic values of buffer levels because of their simplicity and ease of realization [27]. During a production process, the buffer level increases or decreases one by one. We defined a triangle membership function based on the buffer capacity as follows: $[0,0,0.25),(0,0.25,0.5),(0.25,0.5,0.75)$, $(0.5,0.75,1)$, and $(0.75,1,1]$. The number in the membership function multiplies the buffer capacity to get the actual interval of buffer level for linguistic value set $B L$.

The current state of machine $s_{i}(t)$ can be 1 (up) or 0 (down) and consequently has the term set $M S=\{U p, D o w n\}$, which means a machine is at power-on or power-off state.

The output linguistic value of fuzzy rules is taken from the term set $M A=\{$ Strong, High, Medium, Low, Weak\}, which indicates the degree of a machine energy-saving operation. A triangle membership function of the output linguistic value is also adopted based on machine energy-saving tendency in $[0,1]$ as follows: $[0,0,0.25),(0,0.25,0.5),(0.25,0.5,0.75)$, $(0.5,0.75,1)$, and $(0.75,1,1]$. The final output variable of fuzzy logic model $f_{i}(t)$ is a real number between 0 and 1 after defuzzification based on the centre of gravity method.

The second phase of the fuzzy controller is a threshold model for deciding the next state of a machine. If $f_{i}(t)$ is less than $r_{i}$, the final output of the fuzzy controller is to switch the machine into the energy-saving state at the end of its current state during the next time slot. Otherwise, the fuzzy controller gives the machine a power-on signal. The power-on signal refers to two situations. The machine will stay in the work state if its current state is work state, or the machine will be woken up if its current state is energy-saving state. The output of the fuzzy controller is represented as a control signal as follows:

$$
c_{i}(t)=\left\{\begin{array}{ll}
\text { True, } & \text { if } f_{i}(t)<r_{i} \\
\text { False, } & \text { if } f_{i}(t) \geq r_{i}
\end{array},\right.
$$

where True means an energy-saving signal and False means a power-on signal.

\subsection{Fuzzy rules for machine energy-saving operation}

In order to reduce the idle state of a machine, a fuzzy reasoning method is adopted to switch a machine into an energy-saving state (e.g., lower energy state or shutdown state) in advance if the machine has an inclination toward starvation or blockage considering the real-time level of its connected buffers.

In the fuzzy reasoning method, fuzzy logic has a good mathematical framework that integrates human knowledge into the decision procedure. The fuzzy logic makes a system work with practical behaviours by IF-THEN rules, which express the relationship between inputs and outputs with appropriate mathematical representation. The IF-section of a rule is the input that represents conditions under which the rule is applicable. The THEN-section of a rule is the conclusion that will be taken under these conditions. A two-input rule of Mamdani fuzzy logic has the following form:

IF $X$ is A AND $Y$ is B THEN $Z$ is $\mathrm{C}$, 
where $X$ and $Y$ are two input variables, $Z$ is the output variable, and $\mathrm{A}, \mathrm{B}$, and $\mathrm{C}$ have linguistic values, which are fuzzy sets with certain membership functions. The crisp control action is realized by a defuzzification process. For a basic module in Fig. 1, the fuzzy rules can be described as:

$$
\text { IF } b_{j}(t) \text { is } B L^{(g)} \text { AND } b_{l}(t) \text { is } B L^{(g)} \text { AND } s_{i}(t) \text { is } M S^{(g)} \text { THEN } E S_{i}(t) \text { is } M A^{(g)} \text {, }
$$

where $g$ is the rule number $(g=1, \ldots, k) ; i, j$, and $l$ are the numbers of machines or buffers; and $E S$ is the degree of machine energy-saving operation in the next time slot.

Consider that when a machine is not at failure state, the actual levels of its upstream and downstream buffer are represented as $b_{j}^{*}$ and $b_{l}{ }_{l}$ with membership functions $\mu_{B L}^{*}\left(b_{j}^{*}\right)$ and $\mu_{B L}\left(b^{*}\right)$. The degree of energy-saving machine operation at the next discrete time slot is given by:

$$
E S_{i}^{*}=\frac{\sum E S_{i} \mu_{R}^{*}\left(E S_{i}\right)}{\sum \mu_{R}^{*}\left(E S_{i}\right)},
$$

where $\mu_{R}^{*}\left(E S_{i}\right)$ is the membership function of the aggregated operational degree and is given by:

$$
\mu_{R}^{*}\left(E S_{i}\right)=\max _{b_{j}, b_{l}} \min \left[\mu_{A N D}^{*}\left(b_{j}, b_{l}\right), \mu_{F R^{(g)}}\left(b_{j}, b_{l}, E S_{i}\right)\right],
$$

where $\mu_{A N D}^{*}\left(b_{j}, b_{l}\right)$ is the membership function of the inputs, and $\mu_{F R^{(g)}}\left(b_{j}, b_{l}, E S_{i}\right)$ is the membership function of the $g^{\text {th }}$ activated rule. That is,

$$
\begin{gathered}
\mu_{A N D}^{*}\left(b_{j}, b_{l}\right)=\mu_{B L}^{*}\left(b_{j}\right) \wedge \mu_{B L}^{*}\left(b_{l}\right), \\
\mu_{F R^{(g)}}\left(b_{j}, b_{l}, E S_{i}\right)=f_{\rightarrow}\left[\mu_{B L^{(g)}}\left(b_{j}\right), \mu_{B L^{(g)}}\left(b_{l}\right), \mu_{M A^{(g)}}\left(E S_{i}\right)\right]
\end{gathered}
$$

Note that the main control objective is to avoid starvation and/or blockage of the machine by fuzzy reasoning. The controllers will maintain buffers neither full nor empty by switching a machine into an energy-saving state based on the buffer level. When a machine tends to be blocked and/or starved, the controller will make the machine sleep. When a machine tends to block its upstream machine or starve its downstream machine, the controller will wake the machine up.

Table I: Fuzzy rules of the basic 1M2B control module.

\begin{tabular}{|c|c|c|c|}
\hline No. & $\boldsymbol{b}_{j}(\boldsymbol{t})$ & $\boldsymbol{b}_{\boldsymbol{l}}(\boldsymbol{t})$ & $\boldsymbol{E} \boldsymbol{S}_{\boldsymbol{i}}(\boldsymbol{t})$ \\
\hline 1 & Empty & Any & Strong \\
\hline 2 & Almost empty & Empty & High \\
\hline 3 & Almost empty & Almost empty & High \\
\hline 4 & Almost empty & Normal & High \\
\hline 5 & Almost empty & Almost full & Strong \\
\hline 6 & Almost empty & Full & Strong \\
\hline 7 & Normal & Empty & Medium \\
\hline 8 & Normal & Almost empty & Medium \\
\hline 9 & Normal & Normal & Medium \\
\hline 10 & Normal & Almost full & High \\
\hline 11 & Normal & Full & Strong \\
\hline 12 & Almost full & Empty & Weak \\
\hline 13 & Almost full & Almost empty & Low \\
\hline 14 & Almost full & Normal & Medium \\
\hline 15 & Almost full & Almost full & High \\
\hline 16 & Almost full & Full & Strong \\
\hline 17 & Full & Empty & Weak \\
\hline 18 & Full & Almost empty & Low \\
\hline 19 & Full & Normal & Medium \\
\hline 20 & Full & Almost full & High \\
\hline 21 & Full & Full & Strong \\
\hline
\end{tabular}


In our study, 26 fuzzy rules - including 25 rules for $\{U p\}$ state, i.e., $s_{i}(t)=1$, and one rule for machine $\{$ Down $\}$ state, i.e., $s_{i}(t)=0$ - are defined based on the above expert knowledge. The fuzzy rules for $\{U p\}$ state are listed in Table I, where "Any" in the first row of the table means whichever element of the $B L$.

For a general manufacturing system with $N$ basic modules, the fuzzy controller for energy-saving operation will be deployed for each module. The main advantage of this kind of distributed control structure lies in its modularity, which enhances the flexibility of the method. During the implementation phase at the shop floor, the distributed structure is computationally simple, facilitating the real-time application of the whole manufacturing system for energy-saving operation.

\section{SIMULATION STUDY}

In this section, the proposed method is validated by simulation experiments for typical serial and parallel manufacturing systems. The Fuzzy Logic Toolbox of MATLAB and SIMULINK [28] are used as the software tools to build and test all simulations.

In order to make a quantitative evaluation of energy consumption, the following assumptions are used. The machine in work state consumes the working energy $E_{w k}$. The normal idle energy is $E_{i d}$. The machine consumes warm-up energy $E_{w u}$ after it is repaired or after it wakes up from the energy-saving state or shutdown state. The consumed energy of a machine $E_{i}$ and a manufacturing system $E_{s y s}$ in a day shift are calculated as follows:

$$
\begin{gathered}
E_{i}=E_{w k, i} \times T_{w k, i}+E_{i d, i} \times T_{i d, i}+E_{w u, i} \times T_{w u, i} \times\left(N_{f l, i}+N_{e s, i}\right) \\
E_{s y s}=\sum_{i=1}^{n} E_{i}
\end{gathered}
$$

where $T_{w k}, T_{i d}$ and $T_{w u}$ are the time lengths of the corresponding machine states, and $N_{f l}$ and $N_{e s}$ are the numbers of machine failures and energy-saving actions.

\subsection{Case 1: A serial manufacturing system}

A 6M5B serial manufacturing system is a simplified version of a real automotive power-train production line [15]. In order to illustrate the effectiveness of our method and compare the system performance with the method in [15], the same system and energy parameters as in [15] are used in the simulation. The $M T B F$ and MTTR of machines are assumed as exponential distributions. The power rate is assumed to be $0.2 \$ / \mathrm{kWh}$. The simulation is repeated 20 times, and the simulation duration is 3 weeks, i.e., $30240 \mathrm{~min}$.

Three scenarios are examined based on the system performances: baseline production scenario without any energy control (S1); only one machine controlled for energy-saving operation (S2); and multi-machine controlled for energy-saving operation (S3). In baseline scenario $\mathrm{S} 1$, the time lengths of machine states and several measures of system performance are recorded during the simulation experiments. Based on blockage and starvation data of all machines, machine M4 is identified as the system bottleneck [29]. The decision cycle of each machine in S2 and S3 is five times as long as its cycle time.

The main system performances of three scenarios are shown in Table II. In S2, M3 and M5 are solely controlled with the thresholds $r_{3}=0.3$ and $r_{5}=0.3$. In S3, all machines excluding the bottleneck, M4, and the last machine, M6, are controlled at the same time with the thresholds $r_{1}=0.4, r_{2}=0.3, r_{3}=0.2$, and $r_{5}=0.2$. M6 is not controlled in order to maximize system throughput. As shown in Table II, each controlled scenario has a lower energy cost with slight throughput loss compared with the baseline production scenario. 
Table II: System performances of three scenarios.

\begin{tabular}{|c|c|c|c|c|}
\hline Scenario & $\begin{array}{c}\text { Machine } \\
\text { controlled }\end{array}$ & Throughput $[95 \%$ CI] & Energy cost $(\$)[95 \% C I]$ & $\begin{array}{c}\text { Energy cost } \\
\text { per part }(\$)\end{array}$ \\
\hline S1 & - & $3141.5[3110.1,3172.8]$ & $224719.9[223310.8,226129.0]$ & 71.53 \\
\hline \multirow{2}{*}{ S2 } & M3 & $3125.1[3092.1,3158.0]$ & $207919.0[206657.4,209180.5]$ & 66.53 \\
\cline { 2 - 5 } & M5 & $3119.9[3087.9,3151.8]$ & $167465.2[166383.8,168546.7]$ & 53.68 \\
\hline S3 & M1, M2, M3, M5 & $3134.2[3102.0,3166.4]$ & $108394.3[107340.4,109448.1]$ & 34.58 \\
\hline
\end{tabular}

The system performance comparisons are listed in Table III. It is clear that the fuzzy control method is effective in a representative serial manufacturing system, whether one machine or several machines are controlled. The total energy cost is significantly reduced with only slight loss of system throughput, and the energy cost per part is also dramatically cut down. Compared with the method proposed by Zou et al. [15], our method achieves more energy saving and less throughput loss in this case study.

Table III: Comparison of system performances.

\begin{tabular}{|c|c|c|c|}
\hline Method & $\begin{array}{c}\text { \% Reduction in } \\
\text { throughput }\end{array}$ & $\begin{array}{c}\text { \% Reduction in } \\
\text { energy cost }\end{array}$ & $\begin{array}{c}\text { \% Reduction in energy } \\
\text { cost per part }\end{array}$ \\
\hline$[15]$ & $2.70 \%$ & $27.00 \%$ & $24.98 \%$ \\
\hline S2 (M3) & $0.52 \%$ & $7.48 \%$ & $6.99 \%$ \\
\hline S2 (M5) & $0.69 \%$ & $25.48 \%$ & $24.95 \%$ \\
\hline S3 & $0.23 \%$ & $51.76 \%$ & $51.66 \%$ \\
\hline
\end{tabular}

\subsection{Case 2: A manufacturing system with parallel lines}

To illustrate the generality and effectiveness of the proposed method, a more complex system with parallel lines is tested (Fig. 2). There are both machines and buffers in a parallel line, which is different from the parallel machines in $[13,16]$. For the split structure at buffer B2, the system is treated as two basic modules, i.e., B2-M3-B3 and B2-M4-B4. For the merging structure at buffer B5, the system is also decomposed as two basic modules, i.e., B3-M5-B5 and B4-M6-B5.

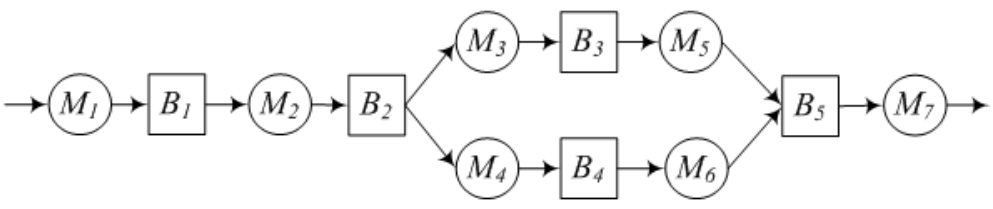

Figure 2: A manufacturing system with parallel lines.

The basic parameters of machines and buffers are listed in Table IV and Table V. In this case, the normal idle energy is assumed to be $40 \%$ lower than its working energy, whereas the warm-up energy of a machine is assumed to be $20 \%$ higher than its working energy [30]. All the simulation scenarios are repeated for 20 replications over an 8-h shift. The decision cycle is $1 \mathrm{~min}$.

Table IV: Reliability and energy-related data of machines.

\begin{tabular}{|c|c|c|c|c|c|c|c|}
\hline Machine & M1 & M2 & M3 & M4 & M5 & M6 & M7 \\
\hline$M T B F(\min )$ & 140 & 70 & 90 & 100 & 150 & 180 & 80 \\
\hline$M T T R(\min )$ & 5 & 20 & 16 & 13 & 3 & 5 & 22 \\
\hline Cycle time (min) & 1 & 1 & 2 & 2 & 2 & 2 & 1 \\
\hline Warm-up (s) & 5 & 3 & 5 & 4 & 6 & 6 & 3 \\
\hline Work energy (kW) & 12 & 5 & 10 & 9 & 17 & 16 & 6 \\
\hline
\end{tabular}


Table V: Buffer capacity and initial level.

\begin{tabular}{|c|c|c|c|c|c|}
\hline Buffer & B1 & B2 & B3 & B4 & B5 \\
\hline Capacity & 12 & 15 & 16 & 16 & 15 \\
\hline Initial level & 7 & 3 & 5 & 4 & 5 \\
\hline
\end{tabular}

After simulating the baseline scenario with no control, M2 is identified as the bottleneck machine. All the machines except M2 and the final machine, M7, are controlled with the thresholds $r_{1}=0.2, r_{3}=0.2, r_{4}=0.2, r_{5}=0.3$, and $r_{6}=0.3$. The energy consumptions of all machines are shown in Fig. 3. It is observed that the energy consumptions of all controlled machines are significantly reduced by about $10 \%$ compared with the baseline scenario. The maximum reduction of machine energy consumption is $18.91 \%$ (observed in M5), and the minimum reduction of machine energy consumption is $9.85 \%$ (observed in M3).

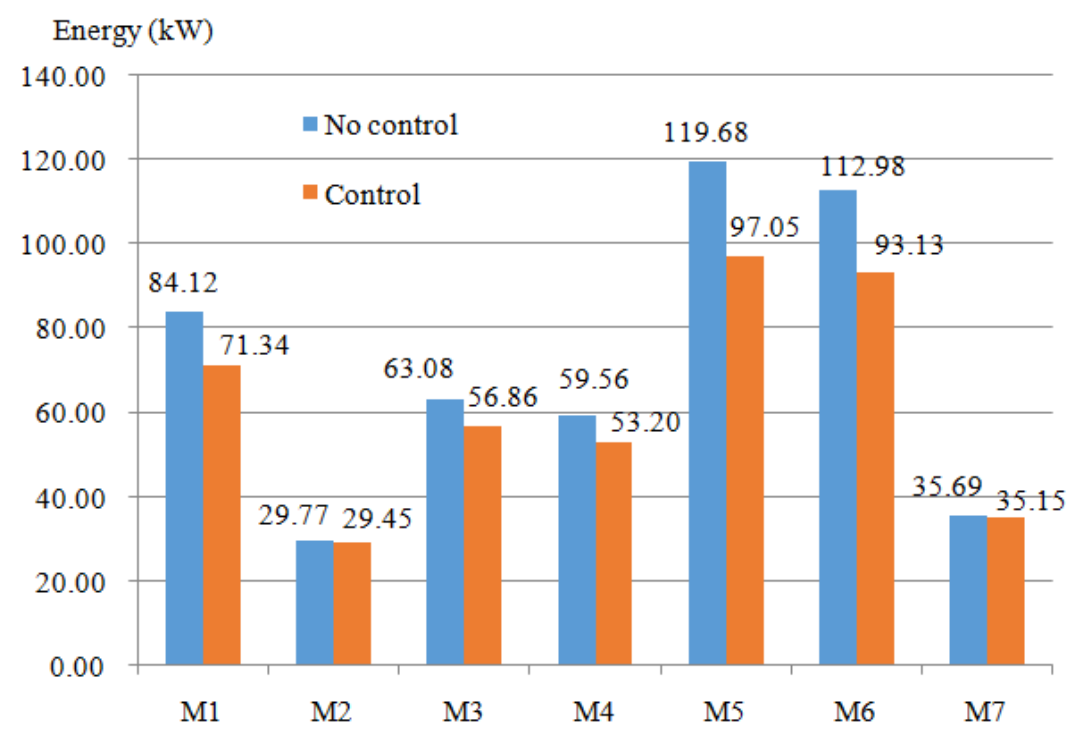

Figure 3: Comparison of machine energy consumption.

The comparison of system performances is shown in Table VI. Through the use of the control method, the system energy consumption exhibits a $13.60 \%$ reduction along with about $3.83 \%$ throughput loss. The energy consumption per part decreases from $1.45 \mathrm{~kW}$ to $1.30 \mathrm{~kW}$, which is $10.34 \%$ reduction. This case illustrates that the fuzzy energy control method is also effective for complex manufacturing systems with parallel lines.

Table VI: Comparison of system performances in case 2 .

\begin{tabular}{|l|c|c|c|}
\hline & $\begin{array}{c}\text { Energy consumption } \\
\text { with 95 \% CI }(\mathrm{kW})\end{array}$ & $\begin{array}{c}\text { System throughput } \\
\text { with 95 \% CI }\end{array}$ & $\begin{array}{c}\text { Energy consumption } \\
\text { per part }(\mathrm{kW})\end{array}$ \\
\hline No control & $504.87[490.40,519.34]$ & $349.00[329.63,368.37]$ & 1.45 \\
\hline Control & $436.21[412.25,460.16]$ & $335.65[315.68,355.62]$ & 1.30 \\
\hline Reduction & $13.60 \%$ & $3.83 \%$ & $10.34 \%$ \\
\hline
\end{tabular}

\section{DISCUSSIONS}

Although machine state control policies have been studied in the literature [8-12], the policies are difficult to define quantitatively. The buffer occupancy is popularly used in production lines for energy control purposes, but the occupancy level has always been controversial in previous research $[12,13,17]$. The main contribution of our study is defining the policies in fuzzy logic for determining the preferred machine states for energy-saving operations. 
Apparently, the fuzzy rules, threshold value, and decision cycle are important to the energysaving control of machines. The influences are discussed quantitatively and qualitatively in this section.

\subsection{Fuzzy rules}

In the first phase of the fuzzy controller (Fig. 1), the fuzzy rules determine the output of the fuzzy reasoning. The output indicates the degree of machine energy saving and takes a real number between zero and one. Obviously, the fuzzy rules imply the knowledge of machine operation for the purpose of saving energy (Table I) and can be defined reasonably by production experts.

According to Eq. (1), the smaller the output of a rule is the more intensive energy-saving operation a machine has. Fig. 4 shows the response surface of the defined fuzzy rules in Table I, where the upstream and downstream buffer levels are normalized with regard to their respective buffer capacities in order to take values between 0 and 1. Fig. 4 shows the effect of the upstream buffer level, $b_{j}$, and the downstream buffer level, $b_{l}$, on the tendency of machine energy-saving action. The filling trend of the downstream buffer or the emptying trend of the upstream buffer will result in smaller $f_{i}$, which will make the machine get into sleep state easier. The emptying trend of the downstream buffer or the filling trend of the upstream buffer will result in larger $f_{i}$, which will make the machine stay in the working state easier. Because the levels of the upstream buffer and downstream buffer change continuously, $f_{i}$ does not keep the same value. Machines will switch their states, and deadlocks can be avoided.

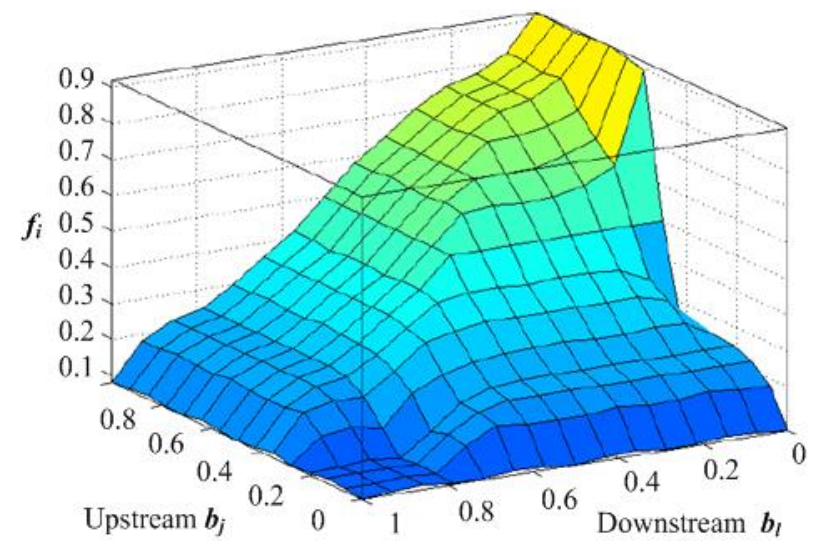

Figure 4: Response surface of the fuzzy rules.

The linguistic value sets, i.e., $B L$ and $M A$, as well as their membership functions, determine the response surface of the fuzzy rules. The number of fuzzy rules depends on the inputs and their element amount of linguistic sets. In this study, five elements in a fuzzy set are used considering the complexity, gradient, and the coverability of the rules. The triangular membership function is used due to the linear change of the buffer level. The optimization of the membership functions will be conducted in future work.

\subsection{Threshold value}

In the second phase of the fuzzy controller, the output of fuzzy reasoning $f_{i}(t)$ will be compared with the threshold value $r_{i}$. Therefore, the threshold value has an important impact on the energy-saving decision. According to Eq. (1), a larger threshold will result in more energy-saving time, whereas a smaller threshold will allow the machine to keep working with less energy-saving time.

The above knowledge is helpful to determine appropriate thresholds for different machines based on their positions in the system. For example, the last machine of the system 
has smaller $r_{i}$ or is not controlled in order to preserve system throughput. The machines near the bottleneck machine will also have smaller $r_{i}$ for the sake of ensuring the productivity of the bottleneck. The system performances under different thresholds in S2 of case 1 are presented in Table VII, where M3 is solely controlled. It is observed that the M3 has more energy-saving time during the production process when $r_{3}$ takes a higher value. More energysaving time results in more loss of machine throughput and system throughput. It should be noted that the optimization of $r_{i}$ is not pursued in this study. In scenario 2 of case 1, the system achieves better performance when the $r_{i}$ of $\mathrm{M} 3$ is equal to 0.2 considering the energy cost per part.

Table VII: Machine and system performances under different $r_{i}$ of M3 in S2.

\begin{tabular}{|c|c|c|c|c|c|c|c|}
\hline $\boldsymbol{r}_{\mathbf{3}}$ & $\mathbf{0 . 2}$ & $\mathbf{0 . 3}$ & $\mathbf{0 . 4}$ & $\mathbf{0 . 5}$ & $\mathbf{0 . 6}$ & $\mathbf{0 . 7}$ & $\mathbf{0 . 8}$ \\
\hline$E S T(\min )$ & 20699.04 & 21002.77 & 21058.08 & 21111.64 & 21162.71 & 21244.92 & 21402.23 \\
\hline$M T P$ & 3147.40 & 3101.55 & 3081.10 & 3061.30 & 3041.70 & 3012.05 & 2952.25 \\
\hline$S T P$ & 3139.95 & 3125.05 & 3122.45 & 3117.40 & 3112.35 & 3101.70 & 3071.10 \\
\hline$E C P(\mathrm{~kW})$ & 66.29 & 66.53 & 66.57 & 66.67 & 66.76 & 66.97 & 67.59 \\
\hline
\end{tabular}

Note: $E S T$ (M3 energy-saving time), MTP (M3 throughput), STP (system throughput), ECP (energy consumption per part)

\subsection{Decision cycle}

The decision of a machine state is based on the change of its connected buffer level. The meaningful minimum decision cycle should equal the machine cycle time. If the decision cycle is much longer than the machine cycle, the decided machine state will last for a longer time in the next decision cycle, and the system will have more throughput loss.

Different decision cycles are also simulated with the same threshold value in case 2 (Fig. 5). The system throughput decreases when a longer decision cycle is used. The largest throughput loss is about $7.18 \%$ of the baseline when the decision cycle is 9 min. Because of the longer control time of the machine, the total system energy consumption also slightly decreases from $436.21 \mathrm{~kW}$ to $423.21 \mathrm{~kW}$ with 1-min and 9-min decision cycles. The energy consumption per part fluctuates irregularly with different decision cycles. A trade-off between energy price and product price should be explored to achieve maximum profit for an appropriate decision cycle in the future. However, setting the length of the machine cycle equal to the length of the decision cycle is a practicable and feasible choice.

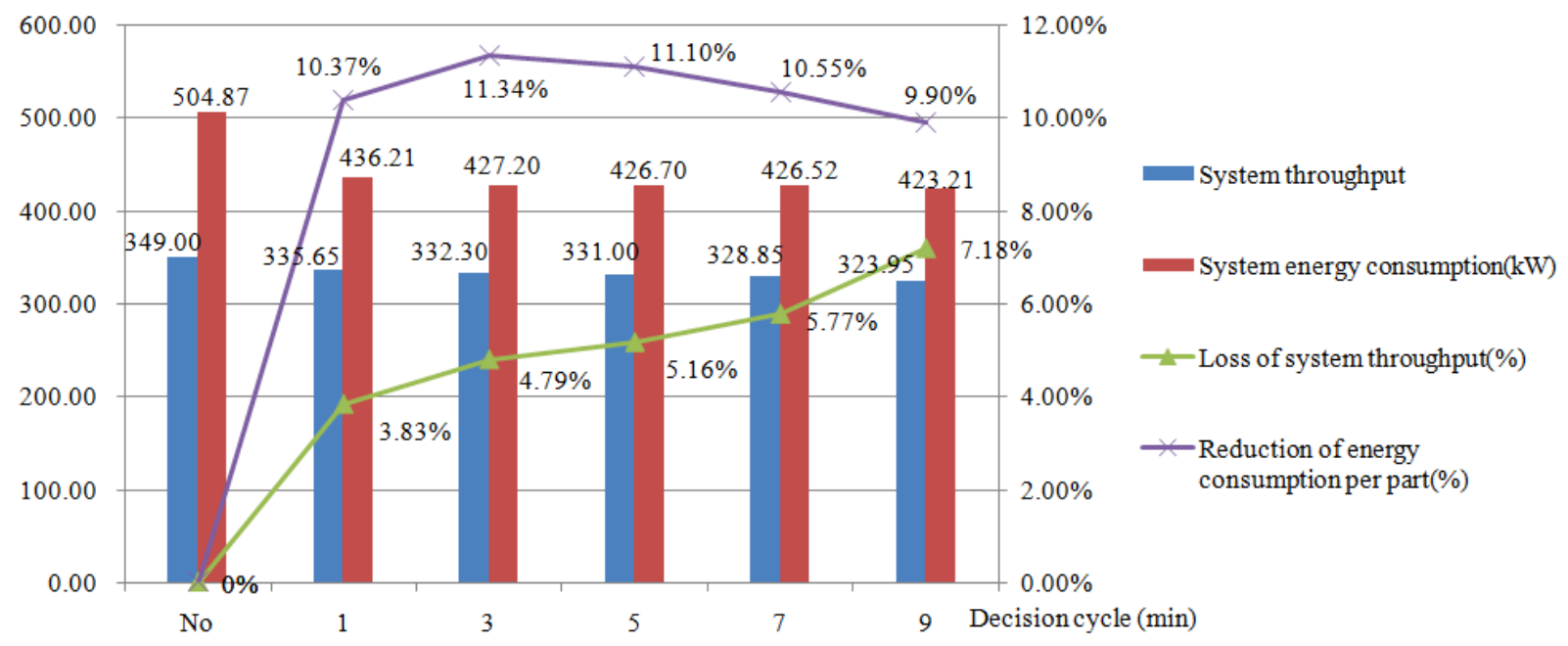

Figure 5: System performance comparison for different decision cycle lengths in case 2. 


\section{CONCLUSION}

In this paper, a fuzzy controller is proposed and developed to reduce the energy consumption of machines in serial and parallel stochastic manufacturing systems. The real-time data of machines and buffers are used as the inputs of the fuzzy controller. The output of the fuzzy logic module determines the degree of a machine's energy-saving operation. The main advantage of the method is that it adopts expert knowledge representation and reasoning. Based on this method, the shop floor manager can choose the target machines to achieve energy-saving control. Single or multi-machine control can be selected flexibly. Numerous simulation experiments are conducted on two manufacturing systems to verify the performance and effectiveness of the proposed method. Compared with the baseline scenario with no energy control, the results show that the proposed control method dramatically decreases energy consumption with acceptable throughput loss. Currently, the machines are distributed controlled based on local real-time data for energy-saving operation. In future work, a supervisory control method will be explored considering real-time system throughput. The extension of this method to more energy-saving states will also be conducted.

\section{ACKNOWLEDGEMENT}

This work was supported by the National Natural Science Foundation of China under Grant number 71571075 .

\section{REFERENCES}

[1] Prabhu, V. V.; Trentesaux, D.; Taisch, M. (2015). Energy-aware manufacturing operations, International Journal of Production Research, Vol. 53, No. 23, 6994-7004, doi:10.1080/ $\underline{00207543.2015 .1100766}$

[2] Duflou, J. R.; Sutherland, J. W.; Dornfeld, D.; Herrmann, C.; Jeswiet, J.; Kara, S., Hauschild, M.; Kellens, K. (2012). Towards energy and resource efficient manufacturing: a processes and systems approach, CIRP Annals, Vol. 61, No. 2, 587-609, doi:10.1016/j.cirp.2012.05.002

[3] Weinert, N.; Mose, C. (2014). Investigation of advanced energy saving stand by strategies for production systems, Procedia CIRP, Vol. 15, 90-95, doi:10.1016/j.procir.2014.06.009

[4] Shrouf, F.; Miragliotta, G. (2015). Energy management based on Internet of Things: practices and framework for adoption in production management, Journal of Cleaner Production, Vol. 100, 235-246, doi:10.1016/j.jclepro.2015.03.055

[5] Chavarria-Barrientos, D.; Batres, R.; Wright, P. K.; Molina, A. (2018). A methodology to create a sensing, smart and sustainable manufacturing enterprise, International Journal of Production Research, Vol. 56, No. 1-2, 584-603, doi:10.1080/00207543.2017.1386333

[6] Hassine, H.; Barkallah, M.; Bellacicco, A.; Louati, J.; Riviere, A.; Haddar, M. (2015). Multi objective optimization for sustainable manufacturing, application in turning, International Journal of Simulation Modelling, Vol. 14, No. 1, 98-109, doi:10.2507/IJSIMM14(1)9.292

[7] Seng, D. W.; Li, J. W.; Fang, X. J.; Zhang, X. F.; Chen, J. (2018). Low-carbon flexible job-shop scheduling based on improved nondominated sorting genetic algorithm-II, International Journal of Simulation Modelling, Vol. 17, No. 4, 712-723, doi:10.2507/IJSIMM17(4)CO18

[8] Mouzon, G.; Yildirim, M. B.; Twomey, J. (2007). Operational methods for minimization of energy consumption of manufacturing equipment, International Journal of Production Research, Vol. 45, No. 18-19, 4247-4271, doi:10.1080/00207540701450013

[9] Prabhu, V. V.; Jeon, H. W.; Taisch, M. (2012). Modeling green factory physics - An analytical approach, Proceedings of the IEEE International Conference on Automation Science and Engineering, 46-51, doi:10.1109/CoASE.2012.6386361

[10] Frigerio, N.; Matta, A. (2015). Energy-efficient control strategies for machine tools with stochastic arrivals, IEEE Transactions on Automation Science and Engineering, Vol. 12, No. 1, 50-61, doi:10.1109/TASE.2014.2344507 
[11] Su, H.; Frigerio, N.; Matta, A. (2016). Energy saving opportunities and value of information: a trade-off in a production line, Procedia CIRP, Vol. 48, 301-306, doi:10.1016/ j.procir.2016.03.147

[12] Jia, Z.; Zhang, L.; Arinez, J.; Xiao, G. (2016). Performance analysis for serial production lines with Bernoulli machines and real-time WIP-based machine switch-on/off control, International Journal of Production Research, Vol. 54, No. 21, 6285-6301, doi:10.1080/00207543.2016.1197438

[13] Chang, Q.; Xiao, G.; Biller, S.; Li, L. (2013). Energy saving opportunity analysis of automotive serial production systems, IEEE Transactions on Automation Science and Engineering, Vol. 10, No. 2, 334-342, doi:10.1109/TASE.2012.2210874

[14] Zou, J.; Arinez, J.; Chang, Q.; Lei, Y. (2016). Opportunity window for energy saving and maintenance in stochastic production systems, Journal of Manufacturing Science and Engineering, Vol. 138, No. 12, Paper 121009, 9 pages, doi:10.1115/1.4033757

[15] Zou, J.; Chang, Q.; Arinez, J.; Xiao, G. (2017). Data-driven modeling and real-time distributed control for energy efficient manufacturing systems, Energy, Vol. 127, 247-257, doi: 10.1016/j.energy.2017.03.123

[16] Li, Y.; Chang, Q.; Ni, J.; Brundage, M. P. (2018). Event-based supervisory control for energy efficient manufacturing systems, IEEE Transactions on Automation Science and Engineering, Vol. 15, No. 1, 92-103, doi:10.1109/TASE.2016.2585679

[17] Sun, Z.; Li, L. (2013). Opportunity estimation for real-time energy control of sustainable manufacturing systems, IEEE Transactions on Automation Science and Engineering, Vol. 10, No. 1, 38-44, doi:10.1109/TASE.2012.2216876

[18] Li, L.; Sun, Z. (2013). Dynamic energy control for energy efficiency improvement of sustainable manufacturing systems using Markov decision process, IEEE Transactions on Systems, Man, and Cybernetics: Systems, Vol. 43, No. 5, 1195-1205, doi:10.1109/TSMC.2013.2256856

[19] Su, W.; Xie, X.; Li, J.; Zheng, L.; Feng, S. C. (2017). Reducing energy consumption in serial production lines with Bernoulli reliability machines, International Journal of Production Research, Vol. 55, No. 24, 7356-7379, doi:10.1080/00207543.2017.1349948

[20] Zhao, R. (2012). Simulation-based environmental cost analysis for work-in-process, International Journal of Simulation Modelling, Vol. 11, No. 4, 211-224, doi:10.2507/IJSIMM11(4)4.218

[21] Tsourveloudis, N. C.; Dretoulakis, E.; Ioannidis, S. (2000). Fuzzy work-in-process inventory control of unreliable manufacturing systems, Information Sciences, Vol. 127, No. 1-2, 69-83, doi: 10.1016/S0020-0255(00)00030-X

[22] Yuniarto, M. N.; Labib, A. W. (2005). Optimal control of an unreliable machine using fuzzylogic control: from design to implementation, International Journal of Production Research, Vol. 43, No. 21, 4509-4537, doi:10.1080/00207540500142969

[23] Tamani, K.; Boukezzoula, R.; Habchi, G. (2011). Application of a continuous supervisory fuzzy control on a discrete scheduling of manufacturing systems, Engineering Applications of Artificial Intelligence, Vol. 24, No. 7, 1162-1173, doi:10.1016/j.engappai.2011.05.017

[24] Wang, J.; Xue, J.; Duque, E. T.; Li, S.; Chang, Q. (2017). Fuzzy decision of machine switch onoff for energy efficient operation of manufacturing system, Proceedings of the $13^{\text {th }}$ IEEE Conference on Automation Science and Engineering, 1158-1162, doi:10.1109/ COASE.2017.8256259

[25] Wang, J.; Fei, Z.; Chang, Q.; Li, S.; Fu, Y. (2019) Multi-state decision of unreliable machines for energy-efficient production considering work-in-process inventory, International Journal of Advanced Manufacturing Technology, 13 pages, doi:10.1007/s00170-018-03213-9

[26] Hajek, P. (1998). Metamathematics of Fuzzy Logic, Kluwer Academic Publishers, Dordrecht

[27] Eker, I.; Torun, Y. (2006). Fuzzy logic control to be conventional method, Energy Conversion and Management, Vol. 47, No. 4, 377-394, doi:10.1016/j.enconman.2005.05.008

[28] Morant, L. (2015). Simulink: Diverse Engineering Applications, Clanrye International, New York

[29] Li, J.; Meerkov, S. M.; Zhang, L. (2010). Production systems engineering: problems, solutions, and applications, Annual Reviews in Control, Vol. 34, No. 1, 73-88, doi:10.1016/ j.arcontrol.2010.02.003

[30] Gutowski, T.; Dahmus, J.; Thiriez, A. (2006). Electrical energy requirements for manufacturing processes, Proceedings of the $13^{\text {th }}$ CIRP International Conference on Life Cycle Engineering, 623-627 